\title{
Plasmonic heating and its possible exploitation in nanolithography
}

\author{
M.B. Cortie, N. Harris, and M.J. Ford, \\ Institute for Nanoscale Technology, University of Technology Sydney, Sydney, NSW, Australia, \\ michael.cortie@uts.edu.au
}

\begin{abstract}
Gold nanoparticles manifest one or more plasmon resonances, resulting in enhanced absorption and scattering of light at the resonant frequencies. The absorbed light is converted to heat. Here we analyze how the resulting localized heat generation might be exploited to generate nanoscale polymer artifacts.
\end{abstract}

Keywords : gold nanoparticle, plasmon resonance, heat transfer models, nanolithography

\section{Introduction}

The optical properties of precious metal nanospheres, nanorods, nanocaps, nanoshells and other nanoscale shapes have attracted much attention recently, e.g. $[1,2]$. This is primarily because the plasmon resonances of these particles are sensitively dependent upon their aspect ratio or shape, allowing for a relatively straightforward way to tune their optical extinction spectra. The longitudinal extinction peak of gold nanorods, for example, may be varied from about $540 \mathrm{~nm}$ to in excess of 1200 $\mathrm{nm}$ by this means[3,4]. The nanoparticles absorb some of the incident light and scatter the rest, with a very small proportion also being reradiated at other wavelengths[5]. The absorbed portion of the light causes heating in the particles[6-10], and it is this phenomenon of 'plasmonic heating'[10] or 'optothermal conversion' [11] that we seek to exploit.

A number of applications can be envisaged for plasmonic heating. In one scheme, a monodisperse population of nanoparticles in colloidal suspension is introduced into an animal or human, is localized at the site of an infection or tumor by active or passive means, and is then illuminated with a suitable light source to deliver its locally destructive payload of heat. This idea has now been demonstrated using nanoshells, nanorods or ordinary nanospheres. We will not discuss it further here and refer the reader to a recent review[12]. A second group of applications relates to solar glazing and spectrally-selective coatings. One option in these products is to block undesired wavelengths of light by absorbing them with plasmonically resonant nanoparticles[13]. Absorptive solar glazing products are not as efficient as reflective ones (e.g.[14]) but may nevertheless enjoy an advantage in terms of economy[15, 16]. In a third possible application, the very high localized heat generation of a nanoparticle undergoing plasmon resonance would be used to modify an underlying or surrounding 'resist' layer or medium, to provide a type of nanoscale lithography. To achieve this, a pattern of nanoparticles might be placed on a mask, or a single nanoparticle could be rastered over the resist, perhaps by attaching it to the tip of a scanning probe microscope. In both cases high intensity illumination at the plasmon resonance frequency is simultaneously applied. It is this idea (Fig. 1) that we will explore here.

We are not the first to have investigated a lithographic scheme of this general nature and, for example, the physico-chemical changes induced in the vicinity of an STM tip undergoing illumination have already received some coverage in the literature[17-20]. Curiously, the emphasis in these schemes has mostly been on exploiting phenomena such as two-photon polymerization, or photocarrier excitation, and the comparatively simple idea of just using the locally raised temperature seems not to have been of much interest prior to our present work. However, we concede that the related concept of a high density memory device based on the polymer-altering action of 
a laser-heated AFM tip certainly has been $\operatorname{mooted}[21]$.

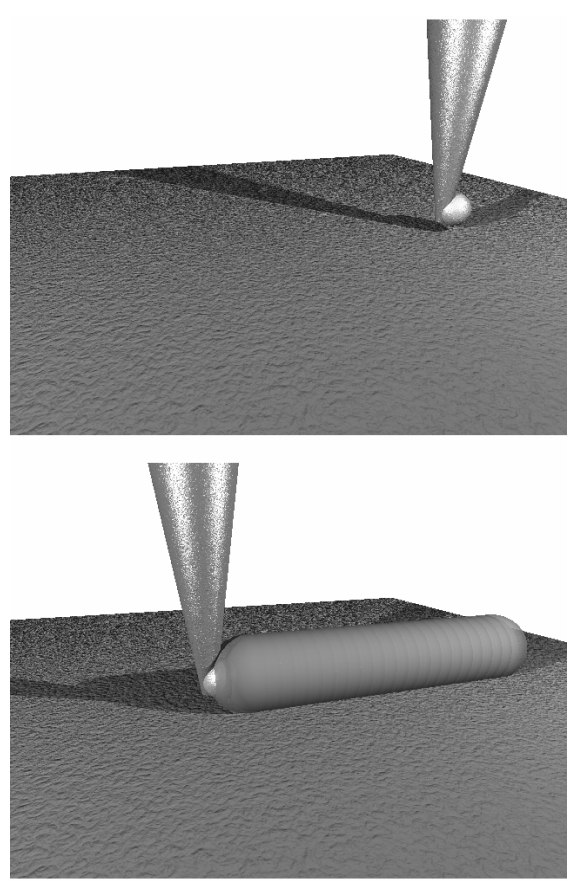

Fig. 1. Schematic illustration of the sub-wavelength fabrication of polymer objects using a guided, plasmonically-heated nanoparticle that polymerizes a nanoscale cylinder of monomer while being swept from right to left and simultaneously illuminated with its resonant wavelength.

\section{Methods}

The fundamental processes involved when nanoparticles convert absorbed photons to thermal energy have been studied and examined in some detail previously e.g. $[5,22-$ 24]. Here we will merely note that, when light interacts with a nanoparticle, it is both absorbed and scattered, with relative proportions depending on the shape, size and composition of the nanoparticle and on the wavelength of the light. Within several femtoseconds the energy of the absorbed photon is manifested in a 'hot' electron distribution. We shall refer to this as the adiabatic stage of the process since heat is not yet transferred to the external environment. However over the next few picoseconds the absorbed energy is converted into lattice phonons [5], which propagate out of the nanoparticle into the surrounding liquid on a time scale varying from tens to hundreds of picoseconds[24] (depending on particle size). A quasi-steady state situation is attained when the heat flow out of the particle equals the rate of generation in the particle. True steady state, when the distribution of temperature in the medium adjacent to the nanoparticle is stable, is only attained some time later.

Under the quasi-steady-state conditions the power absorbed by and transferred out of a particle is given by

$$
\dot{Q}=\int_{\lambda_{1}}^{\lambda_{2}} C_{a b s}(\lambda) E(\lambda) \cdot d \lambda
$$

where $\dot{Q}$ is the heat transfer rate $(\mathrm{W}), C_{\mathrm{abs}}(\lambda)$ the wavelength-dependent absorption cross section, $E(\lambda)$ is the spectral irradiance of the light source $\left(\mathrm{W} \cdot \mathrm{m}^{-2} \cdot \mathrm{nm}^{-1}\right)$, and $\lambda_{1}$ and $\lambda_{2}(\mathrm{~nm})$ are the limiting wavelengths of light over which the light source operates[7]. This heat flow will set up a temperature profile in the surrounding medium.

In the present work we will consider only the situation of a single nanoparticle at temperature $T_{\mathrm{p}}$ immersed in an infinite, conductive medium at initial temperature $T_{\infty}$. Radiant heat transfer can be neglected[8, 22, 25], and for very short periods of irradiation at least, so can convection[7]. At least three heat transfer models for a sphere undergoing heat generation in an infinite, conductive medium are available: a complete solution due to Goldenberg and Trantor[26], a simplified solution due to Pustovalov[8], and a set of solutions for various scenarios by Govorov et al.[6]. The Pustovalov solution is claimed to be valid for $t>t_{\mathrm{T}}$, where $t_{\mathrm{T}}$ is the characteristic time for heat transfer between particle and medium:

$$
t_{T} \approx \frac{a_{0}^{2}}{4 \chi_{m}}
$$

where $\chi_{m}$ is the thermal diffusivity of the medium which we take here as $1.53 \times 10^{-7} \mathrm{~m}^{2} / \mathrm{s}$, the value for water, and $a_{0}$, the particle radius, which we vary between 10 and $40 \mathrm{~nm}$, a practically attainable range for citrate-stabilized gold nanoparticles in an aqueous sol. The applicable $t_{\mathrm{T}}$ for the present model system is $~$ $0.2 \mathrm{~ns}$ for a sphere of $20 \mathrm{~nm}$ diameter and $\sim 0.4$ ns for a $80 \mathrm{~nm}$ diameter sphere. The predictions of the Goldenberg-Trantor (G-T) solution are compared in Fig. 2 to those of Pustovalov for a gold particle of $10 \mathrm{~nm}$ radius. Use of the G-T solution requires the numerical integration of a 
rather poorly behaved function. Here a million steps have been used and the function was integrated between 0 and 30. This was sufficient to produce stable solutions for the present problem. However, the models are in agreement only for times $t>>t_{\mathrm{T}}$. We will use the G-T model in our subsequent calculations here because it is a more complete solution based on fewer simplifications. Note however, that both models assume heat transfer by conduction, and therefore both will seriously underestimate the particle temperature should the surrounding medium vaporize, thereby causing the dominant mechanism of heat transfer to switch to convection[7]. The temperature at which this would occur depends on the radius of the particle, on the time scale and perhaps on the availability of a nucleation site for the steam bubble. In any event it will be above $373 \mathrm{~K}$ and the phenomenon will without doubt always occur above $647 \mathrm{~K}$, the critical temperature of $\mathrm{H}_{2} \mathrm{O}$.

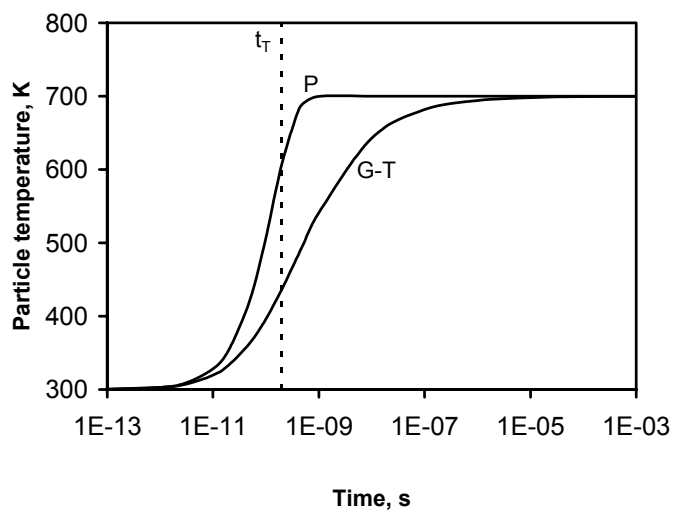

Fig. 2. Comparison of transient heating in a gold nanoparticle of $10 \mathrm{~nm}$ radius in a conductive medium of $\mathrm{H}_{2} \mathrm{O}$ subjected to $521 \mathrm{~nm}$ laser irradiation at $7.19 \times 10^{10} \mathrm{~W} / \mathrm{m}^{2}$. The Pustovalov and Goldenberg-Trantor solutions only converge when $\mathrm{t}>50000 . \mathrm{t}_{\mathrm{T}}$.

Cooling of a particle was calculated using the Cooper solution to a sphere immersed in an infinite conductive medium[27] as well as by using a transient convective solution from the text of Becker[28], with a value of $h$, the heat transfer coefficient calibrated to the measured time-to-half-temperature cooling data of $\mathrm{Hu}$ and Hartland[24]. In both instances, the temperature of the surrounding medium was set at $T_{\infty}$. This might be appropriate for the case of a heat-generating particle attached to a moving probe tip but represents an overestimate of the cooling rate of a sphere immersed in a preheated medium.

Formation of an arbitrary nanoscale pattern in the polymer is a two stage process. First, one or more gold nanoparticles have to be placed in the desired configuration by some means, then a polymer shape around them is generated using plasmonic heating. We will not delve much here into the methods by which the nanoparticles could be positioned, save to note that the processes of spontaneous self-assembly can be manipulated to produce configurations such as long chains of gold nanorods, e.g.[29, 30] while individual nanoparticles can be moved around by an AFM tip[31, 32], a tedious but ultimately completely deterministic process. The important point here is that there will be a target range of temperature in which optimum polymerization of the monomer medium will occur. We will examine the effect of particle geometry, laser fluence and laser intensity on the width and characteristics of this target heat affected zone (HAZ), and seek to establish the conditions under which optimum performance might be expected.

\section{Results}

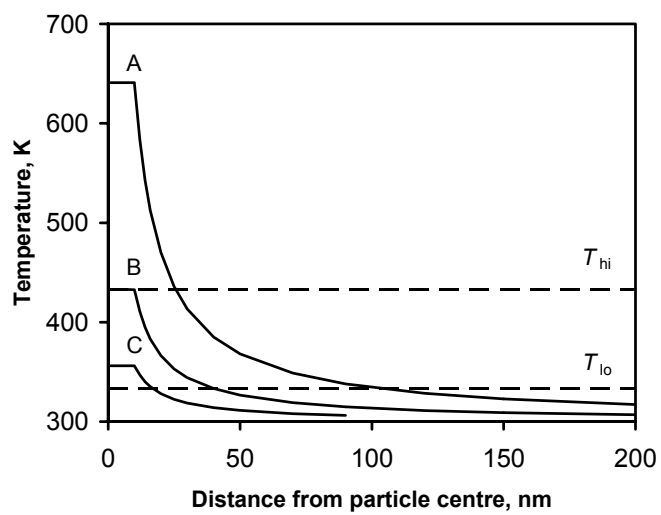

Fig. 3. Illustrative temperature profiles of a $10 \mathrm{~nm}$ particle irradiated with too high an intensity, too low (C) and an appropriate intensity (B).

The depth of the HAZ is of critical importance in respect of lithography, and is very sensitive to experimental conditions, Fig. 3. We will designate the lowest temperature at which polymerization occurs (within the time frame of the experiment) to be $T_{\mathrm{lo}}$. However, 
there is also an upper temperature limit, $T_{\mathrm{hi}}$, above which thermally-induced degradation of the newly-formed polymer would occur (case A in Figure 3). A special situation arises when $T_{\mathrm{p}} \approx T_{\mathrm{hi}}$, - representing the maximum heat generation that can be achieved without destroying the polymer (case B). We will examine this particular case further here, while the scenario where $T_{\mathrm{p}}<T_{\mathrm{lo}}$, is clearly suboptimum and will not be considered further.

For the purposes of the present exercise we assign $T_{\mathrm{lo}}$ and $T_{\mathrm{hi}}$ to be 60 to $160^{\circ} \mathrm{C}$ respectively, giving a usable HAZ of $100 \mathrm{~K}$. These limits appear to be roughly appropriate for a system requiring plasmonically-assisted localized polymerization of a water-soluble monomer. However, there would be no loss in the generality of our analysis if some other range of target temperatures was used.

Calculated heating and cooling curves for gold nanospheres of between 10 and $40 \mathrm{~nm}$ radius, suspended in $\mathrm{H}_{2} \mathrm{O}$ and subjected in each case to laser irradiation at an intensity that will produce a steady-state particle temperature of $T_{\mathrm{p}}=T_{\mathrm{hi}}=433 \mathrm{~K}$ are shown in Fig. 4. Attainment of a narrow heat affected zone and hence high resolution requires use of the smallest practical particle.

The cooling transients calculated using the conductive and convective models are subtly different (cf. Figs. 4(b) and 4(c)). For this system, convective cooling initially maintains temperature of the particle for longer, but is followed by a steeper transient. However, either way, the particles will have cooled to ambient within $1 \times 10^{-8}$ seconds.

The depth of the HAZ turns out to be a linear function of particle diameter for the special case where $T_{\mathrm{p}}=T_{\text {hi }}$, Fig. 5(a). Note however, that a variation in laser intensity is required to ensure this special situation. The resulting HAZ zones are graphically rendered in Fig. 5(b).

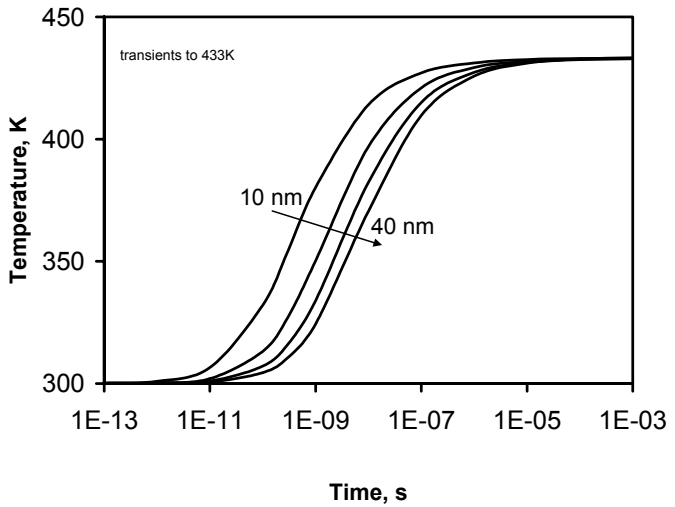

(a)

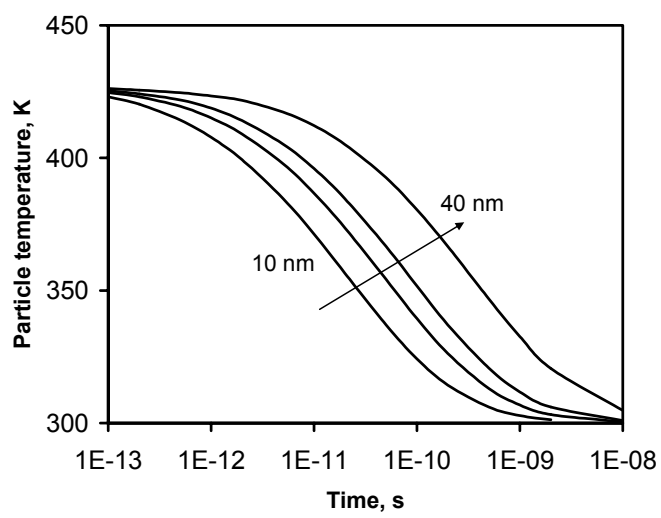

(b)

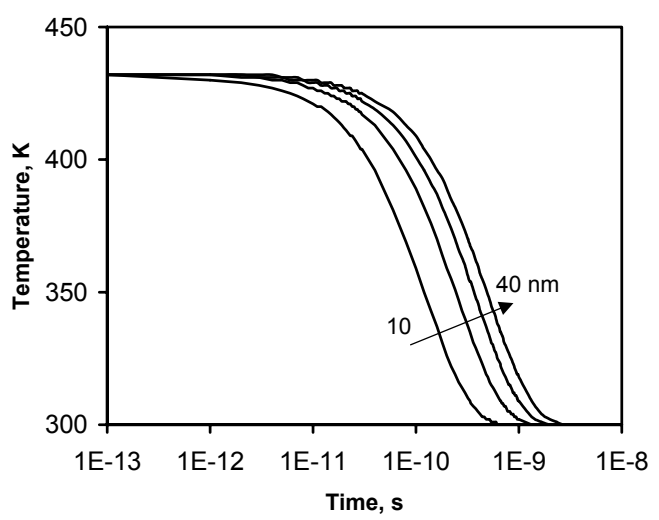

(c)

Fig. 4. (a) Heating up of spheres of different radii subjected to laser illumination to produce a particle temperature of $433 \mathrm{~K}$ at steady-state. (b) Cooling transients in particles after laser irradiation switched off (assumes particle is quenched in conductive medium at $300 \mathrm{~K}$ ). (c) Cooling transients as for (b) but using transient convective heat transfer model. 


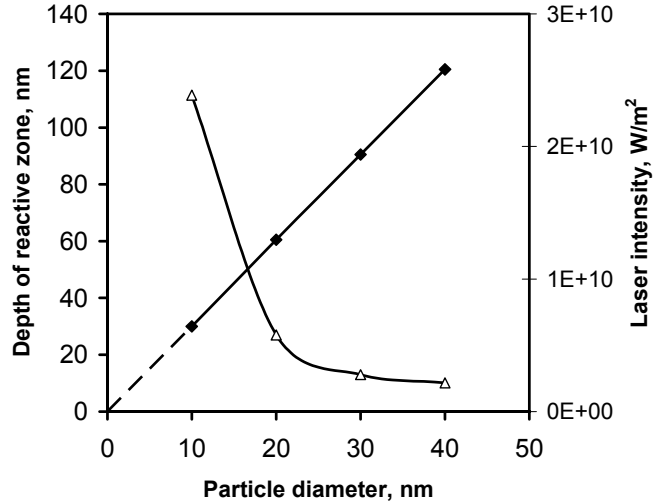

(a)
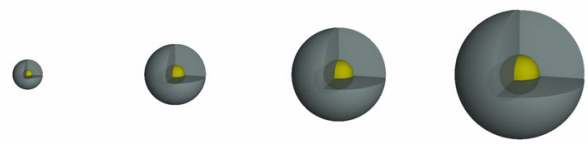

(b)

Fig. 5. (a) Depth of the target heat affected zone as a function of particle diameter and $T_{\infty}$. Note that the irradiation intensity is also varied in these simulations in order to ensure the particle temperature is constant at $433 \mathrm{~K}\left(160^{\circ} \mathrm{C}\right)$. (b) graphical rendering of target heat affected zones of (a).

\section{Conclusions}

Heat will be generated in gold nanospheres undergoing plasmon resonance, and this heat will flow into the surrounding medium to create a heat affected zone (HAZ). An optimum situation is obtained when $T_{\mathrm{p}}=T_{\mathrm{hi}}$. In this case heat-up times would be of the order of $1 \times 10^{-6}$ to $1 \times 10^{-5} \mathrm{~s}$, while cooling would take at least $1 \times 10^{-9} \mathrm{~s}$. In the optimum scenario, the depth of the HAZ is linearly proportional to the size of the heat-generating particles.

This work was supported by the Australian Research Council and the University of Technology Sydney.

\section{References}

1. C. J. Murphy et al., J. Phys. Chem. B 109, pp.13857-13870, (2005).

2. K. L. Kelly et al., J. Phys. Chem. B 107, pp.668677, (2003).

3. Y.-Y. Yu et al., J. Phys. Chem. B 101, pp.66616664, (1997).
4. J. Perez-Juste et al., Coordin. Chem. Rev. 249, pp.1870-1901, (2005).

5. S. Link et al., Inter. Rev. Phys. Chem. 19 (3), pp.409-453, (2000).

6. A. O. Govorov et al., Nanoscale Res Lett, pp.DOI 10.1007/s11671-006-9015-7, (2006).

7. N. Harris et al., J. Phys. Chem. B. 110, pp.10701-10707, (2006).

8. V. K. Pustovalov, Chem. Phys. 308, pp.103108, (2005).

9. S. Link et al., Chem. Phys. Let. 315, pp.12-18, (1999).

10. M. Cortie et al. Smart Materials, Nano-, and Micro-Smart Systems II, Sydney, Australia, 12th15th Dec, 2005; Al-Sarawi, S. F., Ed. SPIE: Sydney, Australia, 2005; pp 565-573.

11. C.-H. Chou et al., J. Phys. Chem. B 109, pp.11135-11138, (2005).

12. D. Pissuwan et al., Trends Biotechnol. 24 (2), pp.62-67, (2006).

13. S. Schelm et al., Appl. Phys. Lett. 82 (24), pp.4346-4348, (2003).

14. H. Chowdhury et al., ASME Journal of Solar Energy Engineering 127, pp.70-75, (2005).

15. X. Xu et al., Chem. Mater. 16 (1), pp.22592266, (2004).

16. X. Xu et al., Gold Bull., in press, (2006).

17. H. Diesinger et al., J. Applied Phys. 90 (9), pp.4862-4864, (2001).

18. S. Grafström, J. Applied Phys. 91 (4), pp.17171753, (2002).

19. F. Cacialli et al., Ultramicroscopy 100, pp.449455, (2004).

20. B. J. Postnikova et al., Microelectronic Engineering 69, pp.459-465, (2003).

21. H. J. Mamin et al., Appl. Phys. Lett. 61 (8), pp.1003-1005, (1992).

22. H. Kurita et al., Appl. Phys. Lett. 72 (7), pp.789-791, (1998).

23. S. Link et al., Physical Review B 61 (9), pp.6086-6090, (2000).

24. M. Hu et al., J. Phys. Chem. B 106, pp.7029 7033, (2002).

25. C. M. Pitsillides et al., Biophys. J. 84, pp.4023 - 4032, (2003).

26. H. Goldenberg et al., Br. J. Appl. Phys. 3, pp.296 - 298, (1952).

27. F. Cooper, International Journal of Heat and Mass Transfer 20 (9), pp.991-993, (1976).

28. M. Becker, Heat Transfer. A Modern Approach. Plenum Press: New York, 1986.

29. K. K. Caswell et al., J. Am. Chem. Soc. 125, pp.13914-13915, (2003).

30. K. G. Thomas et al., J. Phys. Chem. B 108, pp.13066-13068, (2004).

31. S. Hsieh et al., J. Phys. Chem. B 106 (2), pp.231-234, (2002).

32. S. A. Maier et al., Adv. Mater. 13 (19), pp.1501-1505, (2001). 